\title{
Interaction Between Stimulated Raman Scattering and Ion Acoustic Waves in Ignition Relevant Plasmas
}

\author{
R. K. Kirkwood, B. J. MacGowan, D. S. Montgomery, B. B. Afeyan, \\ W. L. Kruer, J. D. Moody, K. G. Estabrook, C. A. Back, S. H. Glenzer, \\ M. A. Blain, E. A. Williams, R. L. Berger, B. F. Lasinski
}

June 1, 1997

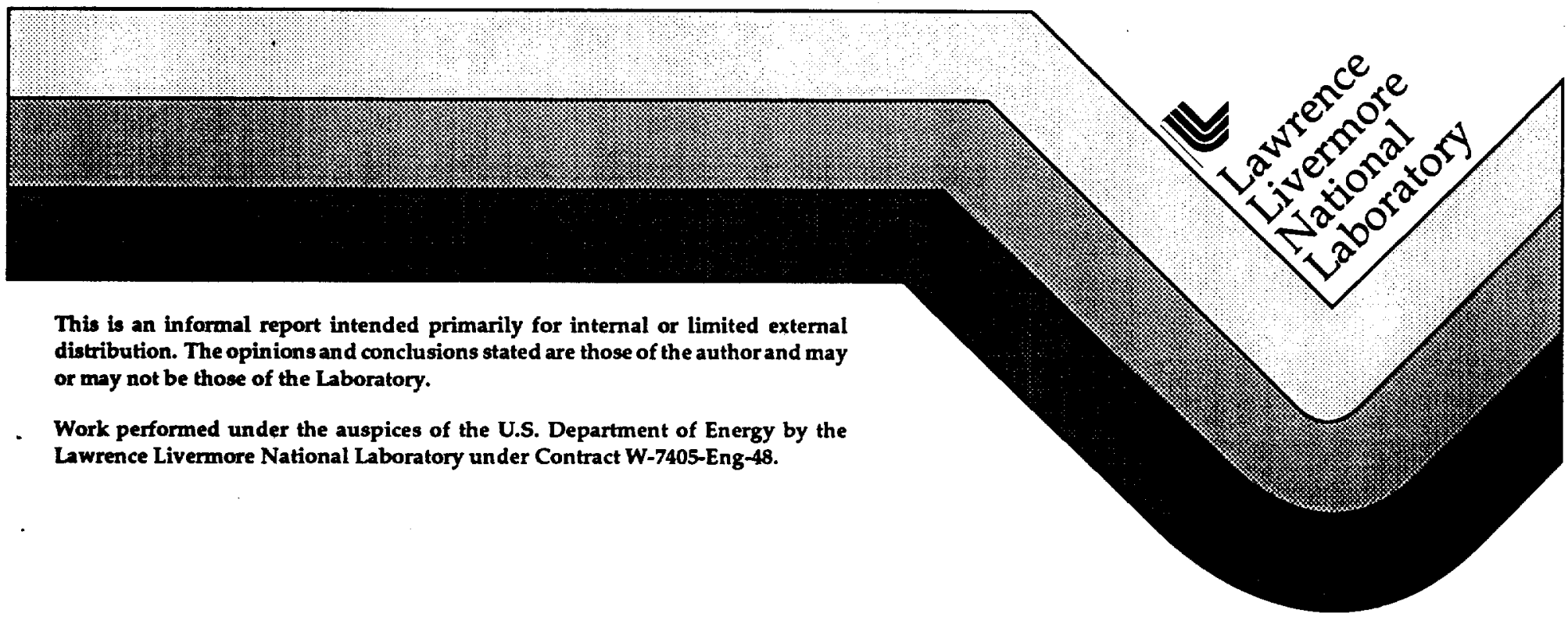




\section{DISCLAIMER}

This document was prepared as an account of work sponsored by an agency of the United States Government. Neither the United States Government nor the University of California nor any of their employees, makes any warranty, express or implied, or assumes any legal liability or responsibility for the accuracy, completeness, or usefulness of any information, apparatus, product, or process disclosed, or represents that its use would not infringe privately owned rights. Reference herein to any specific commercial product, process, or service by trade name, trademark, manufacturer, or otherwise, does not necessarily constitute or imply its endorsement, recommendation, or favoring by the United States Government or the University of Califormia. The views and opinions of authors expressed herein do not necessarily state or reflect those of the United States Government or the University of California, and shall not be used for advertising or product endorsement purposes. 
Interaction Between Stimulated Raman Scattering and Ion Acoustic Waves In Ignition Relevant Plasmas

\author{
R. K. Kirkwood, B. J. MacGowan, D. S. Montgomery, B. B. Afeyan, \\ W. L. Kruer, J. D. Moody, K. G. Estabrook, C. A. Back, S. H. Glenzer, \\ M. A. Blain ${ }^{\dagger}$, E. A. Williams, R. L. Berger, and B. F. Lasinski \\ Lawrence Livermore National Laboratory, University of California, \\ L-473 P.O. Box 808, Livermore California 94550, U.S.A. \\ tCentre D'Etudes de Limeil-Valenton, France.
}

\begin{abstract}
We have observed that the scattering of light by stimulated Langmuir waves (SRS) in ignition scale plasmas is dependent on the properties of the ion acoustic wave, indicating that a nonlinear coupling between the two waves limits the scattered energy
\end{abstract}




\section{Introduction}

The transport of intense laser beams through large regions of under dense plasmas is important to the achievement of indirect drive inertial confinement fusion (ICF) [1]. The energy deposition profile of the laser beam in the target is strongly affected by stimulated scattering from ion acoustic, and Langmuir waves in the plasma created when the target material ionizes. These interactions are most simply modeled by the three wave decay processes of stimulated Brillouin scattering (SBS) and stimulated Raman scattering (SRS), respectively [2]. The three wave decay models assume that waves propagating in the plasma do not interact, that is, that the plasma response to the ponderomotive force of the incident and scattered light is linear. However, previous experimental observations $[3,4]$ and theoretical studies [5-13] provide evidence that the Langmuir waves and the ion acoustic waves are not independent. In fact, in the high temperature, high $\mathrm{Z}$ plasmas present in indirect drive targets [1] secondary decay instabilities can limit the SRS [9-13]. In these secondary processes the Langmuir wave produced by the SRS process can itself decay into an ion acoustic wave and either a secondary Langmuir wave (LDI) or a secondary electromagnetic wave (EDI), thus coupling the properties of the Langmuir wave to the properties of the ion acoustic wave directly. The mechanism for limiting SRS reflectivities by LDI was first described by Karttunen [9] and Heikkinen and Karttunen [10], and was later invoked to explain the SRS spectrum from Au foil plasmas [11]. These processes were subsequently shown to occur in numerical simulations $[7,8,12]$. Recently Baker [13] suggested that the EDI may also result in a limit to the reflectivity.

In this article we describe the first demonstration that the SRS reflectivity in a plasma can depend directly on ion wave damping, and find 
that the SRS reflectivity in our case is consistent with Langmuir wave amplitudes that are limited by a secondary decay. The experiments are done in a low density $\mathrm{Xe}$ gas target plasma, in which $\mathrm{C}_{5} \mathrm{H}_{12}$ is added as a low $\mathrm{Z}$ impurity [14]. Measurements of x-ray spectra and transmitted light, and calculations all show that variation of the concentration of impurities below $10 \%$ has very little effect on the electron density, temperature, or collisional absorption rate, while calculations indicate the impurities have a large affect on the ion wave damping. The SRS reflectivity, or equivalently the Langmuir wave amplitude, is found to depend on the impurity concentration and thus on ion wave damping, and to track the threshold for decay of the Langmuir wave. The plasmas produced in these experiments mimic the properties of the plasma that forms near the wall in an Au hohlraum filled with a low $\mathrm{Z}$ gas such as will be used in ignition experiments on the National Ignition Facility (NI) at somewhat lower laser intensity [1], while providing a uniform and well characterized plasma. Other experiments in gas filled hohlraums have also shown a dependence of SRS on ion wave damping both when the wall is doped with a Be impurity to mimic the gradients and time dependence of the wall plasma expected in NIF experiments [15], and when the gas has large fractions of both low and high $\mathrm{Z}$ material [16]. However plasma properties vary along the ray path in those experiments making identification of the physical mechanism difficult.

\section{Experimental Design}

The experiments were carried out on the Nova laser facility in an approximately spherical plasma produced by nine $f / 4.3, \lambda=351 \mathrm{~nm}$ beams. These heater beams each produce 2.5 TW of power continuously for $1 \mathrm{~ns}$, with a total power of 22.5 TW. The beams pass through a gas mixture at 1 
atm. of pressure contained inside a $260 \mathrm{~nm}$ thick spherical polyimide shell with radius $r_{0}=1.3 \mathrm{~mm}$ [17]. The heater beams are aligned to cross at the center of the target. The heaters are defocused such that they are converging with a beam radius that is approximately equal to the target radius, providing spatially uniform heating. The electrons are heated to a temperature of $\mathrm{T}_{e}=$ $3.6 \mathrm{keV}$ during the $\mathrm{t}=0.5$ to $1.0 \mathrm{~ns}$ period when the plasma parameters are most constant in space and time [17]. The electron density is determined by the initial gas density and the average charge state. At this temperature $\mathrm{Xe}$ has a charge state of about 40 [18], leading to a density of $n=8.5 \times 10^{20} \mathrm{~cm}^{-3}$. Since the $\mathrm{C}_{5} \mathrm{H}_{12}$ impurity is fully ionized and carries 42 electrons per molecule, the electron density is independent of its concentration to within $\leq$ $5 \%$. The temperature is determined from measurements of $x$-ray line ratios and X-ray transport modeling as described in Ref. [18] and is in agreement with LASNEX [19] simulations. Measurements of radiated power and beam attenuation by collisional absorption indicate that the temperature is also insensitive to impurity concentration. The measured transmission of a beam through the plasma [20] varies only from $1 \%$ to $2 \%$ when the impurity concentration is varied from $0 \%$ to $30 \%$ and is also in agreement with the simulations, similarly indicating that the electron temperature variations are small $(\leq \pm 6 \%)$. Measurements of the radiated $x$-ray power in the photon energy range of 0.2 to $2 \mathrm{keV}$ [21] indicate that the radiated power is also constant within $\pm 15 \%$ over the same range of impurity concentration. The lack of dependence of the electron density and temperature on impurity concentration indicates that the frequency and damping rate of the Langmuir wave are also constant.

An interaction beam, produces 1.5 TW continuously for 1.0 ns with $\lambda=$ $351 \mathrm{~nm}$. This beam is delayed $0.5 \mathrm{~ns}$ with respect to the heaters and focused at 
the plasma center. Reflectivity measurements are made during the 0.5 to 1.0 ns (early) period when the heaters are on, as well as during the 1.0 to $1.5 \mathrm{~ns}$ (late) period when the plasma is cooling and less homogeneous. The interaction beam is smoothed by a random phase plate (RPP) and $0.7 \AA$ FM bandwidth dispersed across the beam in the near field (SSD), so that its peak intensity and spot size in vacuum are $7.0 \times 10^{15} \mathrm{~W} / \mathrm{cm}^{2}$ and $177 \mu \mathrm{m}$ FWHM ( $345 \mu \mathrm{m}$ between first Airy minima). The plasma properties encountered by the interaction beam during the early period are calculated by LASNEX [19] for a $90 \% \mathrm{Xe}_{1}, 10 \% \mathrm{C}_{5} \mathrm{H}_{12}$ gas mix, indicating a temperature, and density plateau near the plasma edge as shown in Figure 1. The classical collisional absorption length for $351 \mathrm{~nm}$ light in Xe with a $3.6 \mathrm{keV}$ electron temperature and $8.5 \times 10^{20} \mathrm{~cm}^{-3}$ electron density, is $800 \mu \mathrm{m}$. Therefore, the majority of the back scattering occurs outside $r=0.5 \mathrm{~mm}$. The down shifted light scattered within $20^{\circ}$ of direct backscatter is measured [22] with a streaked optical spectrometer in the visible, with a spectral range of 400 to $700 \mathrm{~nm}$, and a second spectrometer in the UV, with a spectral range of 346 to $361 \mathrm{~nm}$. The SRS light detected by the long wavelength spectrometer from an experiment with $5.5 \% \mathrm{C}_{5} \mathrm{H}_{12}$ impurity is shown in figure 2 . During the early period the peak of the spectrum is at $575 \mathrm{~nm}$ consistent with scattering from a Langmuir wave propagating in a plasma with a density equal to $10 \%$ of the critical density and a $3.0 \mathrm{keV}$ electron temperature comparable to the simulated plasma parameters near $r=1.2 \mathrm{~mm}$. After the heaters turn off at $1.0 \mathrm{~ns}$ the peak shifts to the blue indicating cooling and expansion of the plasma.

\section{Observations of Scattering verses Ion Wave Damping}

Experiments were performed with six different impurity concentrations between $0 \%$ and $30 \% \mathrm{C}_{5} \mathrm{H}_{12}$ and exhibit a strong dependence 
of the SRS reflectivity on the concentration of $\mathrm{C}_{5} \mathrm{H}_{12}$ which is interpreted as a dependence of Langmuir wave amplitude on the damping rate of the ion acoustic wave. The integrated energies from the two time periods are expressed as percent reflectivities of the incident beam power due to SRS and plotted in Figure 3. A similar analysis has been done for back scattered light between $350.5 \mathrm{~nm}$ and $352 \mathrm{~nm}$, which is interpreted as SBS back scatter and plotted in Figure 4. In the integrated data it is clear that late time SRS reflectivities are approximately proportional to the impurity concentration for all concentrations studied. The early time reflectivities are proportional to concentration up to $10 \%$ and become independent, or a mildly decreasing function, of concentration between 10 and $30 \%$. Because the ion acoustic damping rate is expected to be linear with impurity concentration in this case [23] a linear dependence of reflectivity on impurity concentration is interpreted as a linear dependence on the damping of the ion acoustic wave. The possibility that dependence of the reflectivity on ion wave damping is actually the result of suppression of SRS by large amplitude ion waves generated by SBS (as discussed in Ref.s [3,4] and references therein), is unlikely because of the observed dependence of SBS on ion wave damping is weak in this case [14]. These observations are the first demonstration of the direct dependence of SRS reflectivity on the damping rate of the ion acoustic wave. In particular the early time reflectivity for concentrations between $1 \%$ and $10 \%$, demonstrates SRS increasing with ion wave damping when the electron-ion collision rate, electron temperature, radiated power, and SBS level are essentially constant.

\section{Comparison with Models of Secondary Decays involving Ion Waves}


A simple model is presented to show how the observed dependence of the reflectivity on ion wave damping may be explained by secondary decay of the Langmuir wave. In this model the intensity profile of the beam and filamentation are neglected for simplicity, and the Langmuir wave amplitude is assumed to be in the vicinity of the threshold for the secondary decay. This threshold for decay, expressed in terms of the density fluctuation, is given by Ref. [9] for LDI and by Ref. [13] for EDI.

$$
\begin{aligned}
& \left(\frac{\delta \mathrm{n}}{\mathrm{n}}\right)_{\mathrm{EDI}}=4 \mathrm{k}_{\mathrm{L}} \lambda_{\mathrm{D}}\left(\frac{v_{\mathrm{ia}}}{\omega_{\mathrm{ia}}}\right)^{1 / 2}\left(\frac{v_{\mathrm{em}}}{\omega_{\mathrm{p}}}\right)^{1 / 2} . \\
& \left(\frac{\delta \mathrm{n}}{\mathrm{n}}\right)_{\mathrm{LDI}}=4 \mathrm{k}_{\mathrm{L}} \lambda_{\mathrm{D}}\left(\frac{v_{\mathrm{ia}}}{\omega_{\mathrm{ia}}}\right)^{1 / 2}\left(\frac{v_{\mathrm{L}}}{\omega_{\mathrm{p}}}\right)^{1 / 2}
\end{aligned}
$$

Where $k_{L}$ is the wave vector of the SRS generated Langmuir wave, $v_{\mathrm{ia}} / \omega_{\mathrm{ia}}$ is the normalized linear ion wave damping rate, $v_{\mathrm{em}}$ is the linear damping rate of the secondary electromagnetic wave and $v_{\mathrm{L}}$ is the linear damping rate of the secondary Langmuir wave, and $\lambda_{D}$ is the electron Debye length. The measured SRS light is interpreted as Thomson scattering off density fluctuations in the scattering volume using the following assumptions. For these experiments the rapid collisional absorption rate of the incident and reflected light determines the size of the interaction region that can be viewed by the back scatter diagnostic. The effective length $L$ used is the depth at which the attenuation of an incident $351 \mathrm{~nm}$ light times the attenuation of the reflected $580 \mathrm{~nm}$ light is equal to $1 / \mathrm{e}$. The length $\mathrm{L}$ is $300 \mu \mathrm{m}$ in this case indicating that is observed SRS comes primarily from the plasma at radii larger than $1 \mathrm{~mm}$. For perfectly coherent fluctuations the reflectivity is proportional to this length to the second power [10], however the finite 
spectral width observed in figure $2\left(\Delta \mathrm{k} \sim 5.6 \times 10^{5} \mathrm{~m}^{-1}\right)$ indicates that in this experiment the maximum distance over which the radiation can be coherent is given by $\ell_{c_{\max }}=1 / \Delta \mathrm{k}$. A finite correlation length will reduce the reflectivity by a factor of at least $\ell_{\mathrm{cmax}} / \mathrm{L}[8]$ below the value for coherent fluctuations, giving the maximum reflectivity for a uniform beam as

$$
\mathrm{R}_{\max .}=\frac{1}{4}\left(\frac{\mathrm{n}}{\mathrm{n}_{\mathrm{co}}}\right)^{2} \mathrm{k}_{0}{ }^{2} \mathrm{~L}(\Delta \mathrm{k})^{-1}\left(\frac{\delta \mathrm{n}}{\mathrm{n}}\right)^{2}
$$

where $k_{0}$ and $n_{c 0}$ are the wave number and critical density of the incident beam. This model indicates that the SRS reflectivity is linearly proportional to ion wave damping if the Langmuir wave amplitude does not grow significantly above the secondary decay threshold. This linear dependence is most clearly observed in the reflectivity data taken at early time when the impurity concentration is less than $10 \%$. For impurity concentrations above $10 \%$ the early time reflectivity is not very dependent on the ion wave damping. This is likely due the convective saturation of the SRS generated Langmuir wave before it reaches the secondary decay threshold. The secondary decay mechanism will only determine the SRS reflectivity when the primary three wave process is sufficiently strong to drive the Langmuir wave amplitude to the threshold for the secondary decay. Thus at early time and high impurity concentration ( $>10 \%$ ) the secondary decay threshold is sufficiently high that the SRS reflectivity is likely limited by the convective saturation of the three wave SRS process and is therefore observed to be approximately independent of ion wave damping. The late time data shows linear dependence of the reflectivity on the impurity concentration up to much higher concentration. This may be because at late time the plasma has cooled and therefore has a higher convective saturation level for the three 
wave process, resulting in reflectivities that follow the linear scaling with ion wave damping up to at least $30 \%$, as shown in figure 3 . However quantitative analysis of the late time data is complicated by variations of the plasma properties in time and space.

As an additional check of the model of secondary decay, the density perturbation is estimated from the measured reflectivity at early time using Eq. 2, and is compared with that predicted by Eqs. 1a,b. To evaluate Eqs 1a,b the damping rates [24] of the Langmuir and electromagnetic waves are calculated using the distribution function expected in a high $\mathrm{Z}$ plasma illuminated with high intensity light [25]. The thresholds are evaluated assuming the average beam intensity $\left(3.0 \times 10^{15} \mathrm{~W} / \mathrm{cm}^{2}\right)$ exists thoughout the interaction region, and are shown as solid and dashed lines in figure 3. In this case the equilibrium distribution is a superGaussian with $n=3.45$ (where $\mathrm{n}=2$ corresponds to a Maxwellian). The estimate shown for the EDI threshold is not changed significantly by inhomogeneities in the beam profile (ie; hot spots), but the estimate for LDI may be significantly reduced by inhomogeneities. This reduction results from the fact that the Langmuir wave Landau damping is strongly sensitive to intensity, as discussed in Ref. [24], so that in a hot spot both the threshold density fluctuation will be much lower, and the gain of the three wave SRS process will be much higher. As a result in a non-uniform beam LDI may be significantly affecting the SRS even when the reflectivity is well below the uniform beam threshold. Comparison of these estimates with data indicates that the average fluctuation amplitudes observed in the experiment at early times and when the impurity concentration is between 0 and $10 \%$ are large enough to excite EDI over much of the beam profile and LDI in intense regions of the beam. 


\section{Conclusions}

We have observed that the SRS reflectivity in a high $Z$ plasma is dependent on the ion wave damping when the Langmuir wave properties are held constant, implying that SRS is limited by a process involving ion acoustic waves. Furthermore, the observed reflectivities are linear with ion wave damping as expected when the electron wave amplitude is limited by EDI or LDI, and the observed wave amplitudes are near or above the thresholds for those instabilities, indicating that the SRS reflectivity in NIF is likely to be limited by these secondary decay processes.

\section{Acknowledgments}

The authors gratefully acknowledge conversations with D. F. DuBois (LANL), W. Rozmus (U. Alberta), R. P. Drake (LLNL), and K. L. Baker (U.C. Davis) on the nature of the LDI and EDI instabilities and acknowledge suggestions by D. Munro (LLNL) and B. H. Wilde (LANL) concerning LASNEX modeling. Work performed under the auspices of the U.S. Department of Energy by the Lawrence Livermore National Laboratory under Contract W-7405-Eng-48.

\section{References}

[1] J. Lindl, Phys. of Plasmas 2, 3933 (1995).

[2] W. L. Kruer, "The Physics of Laser Plasma Interactions", Addison-Wesley Publishing Co. Redwood City Ca.

[3] C. J. Walsh, D. M. Villeneuve, and H. A. Baldis, Phys. Rev. Lett. 53, 1445 (1984).

[4] H. A. Baldis, et. al., Phys. Rev. Lett. 62, 2829 (1989).

[5] H. A. Rose, D. F. DuBois, and B. Bezzerides, Phys. Rev. Lett. 58, 2547 (1987). 
[6] K. Estabrook, W. L. Kruer, and M. G. Haines, Phys. Fluids B 1, 1282 (1989).

[7] T. Kolber, W. Rozmus, and V. T. Tikhonchuk, Phys. Fluids B 5, 138 (1993).

[8] T. Kolber, W. Rozmus, V. T. Tikhonchuk, Phys. Plasmas 2, 256 (1995).

[9] S. J. Karttunen, Phys. Rev. A 23, 2006 (1981).

[10] J. A. Heikkinen and S. J. Karttunen, Phys. Fluids 29, 1291 (1986).

[11] R. P. Drake, and S. H. Batha, Phys. Fluids B 3, 2936 (1991).

[12] B. Bezzerides, D. F. DuBois, and H. A. Rose, Phys. Rev. Lett. 70, 2569 (1993).

[13] K. L. Baker, Ph.D. Dissertation, University of California, Davis (1996), see also P. K. Shukla, et. al., Phys. Rev. A 27, 552 (1983).

[14] R. K. Kirkwood et. al. Phys. Rev. Lett. 77, 2706 (1996).

[15] R. K. Kirkwood to appear in Physics of Plasmas Vol. 4, (1997).

[16] J. C. Fernandez et. al. Phys. Rev. Lett. 77, 2702 (1996).

[17] D. H. Kalantar, et. al., Phys. of Plasmas 2, 3161 (1995).

[18] C. A. Back et. al., to be published

[19] G. Zimmerman and W. Kruer, Comments in Plasma Phys. and Controlled Fusion 2, 85 (1975).

[20] J. D. Moody et. al., Bull. Am. Phys. Soc. 39, 1753 (1994).

[21] H. N. Kornblum, R. L. Kauffman, and J. A. Smith, Rev. Sci. Inst., 57, 2179 (1986).

[22] R. K. Kirkwood et. al. Bull. Am. Phys. Soc. 39, 1753 (1994) and submitted to Rev. Sci. Inst.

[23] E. A. Williams et. al., Phys. of Plasmas 2, 129 (1995).

[24] B. B. Afeyan, A. E. Chou, and W. L. Kruer, submitted to Phys. Rev. Lett. and in this report.

[25] P. Alaterre, J.-P. Matte and M. Lamoureaux, Phys. Rev. A 34, 1578 (1986). and A. B. Langdon, Phys. Rev. Lett. 44, 575 (1980). 
Figure Captions

Fig. 1 Calculated electron density, temperature, and averaged ionization state, at $t=0.7 \mathrm{~ns}$ indicate density and temperature scale lengths are long.

Fig. 2 a) A streaked spectrum of the SRS reflectivity from a target with $5.5 \%$ impurity concentration shows a narrow line consistent with a flat density profile before $1.0 \mathrm{~ns}$ and a decreasing density after $1.0 \mathrm{~ns}$. b) Time integrated reflectivity from a shows a narrow peak at $575 \mathrm{~nm}$ in the 0.5 to $1.0 \mathrm{~ns}$ 'early' period. c) The spectrally integrated power collected by the SRS and SBS detectors is shown for the case in a).

Fig. 3 SRS reflectivities averaged over the $0.5 \mathrm{~ns}$ to $1.0 \mathrm{~ns}$ 'early' period and the $1.0 \mathrm{~ns}$ to $1.5 \mathrm{~ns}$ 'late' period are shown vs. impurity concentration. The impurity concentration is interpreted as the ion wave damping rate and the reflectivity is interpreted as the square of the fluctuation amplitude. Solid and dashed lines represent the EDI and LDI threshold amplitudes for a uniform beam and parameters relevant to early time data. The LDI limited reflectivity of in a non-uniform beam can be much lower than what is shown as discussed in the text. 


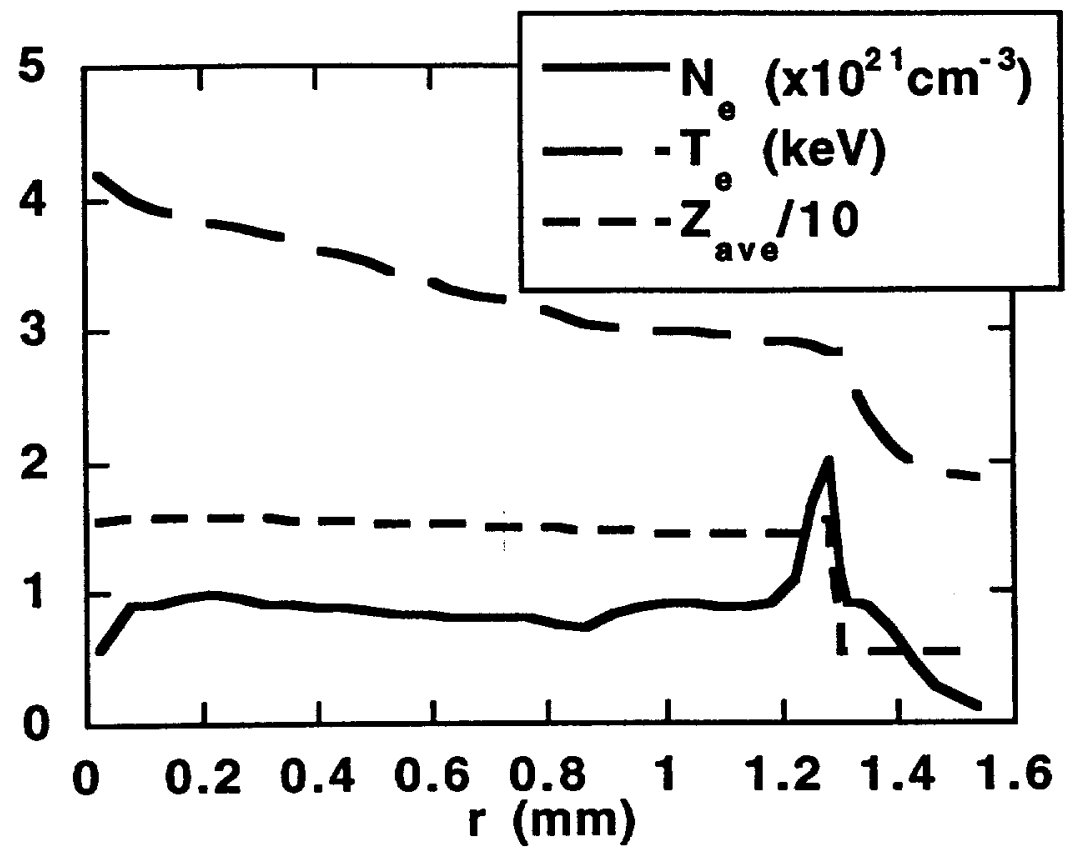

Figure 1 
Figure 2(a) 

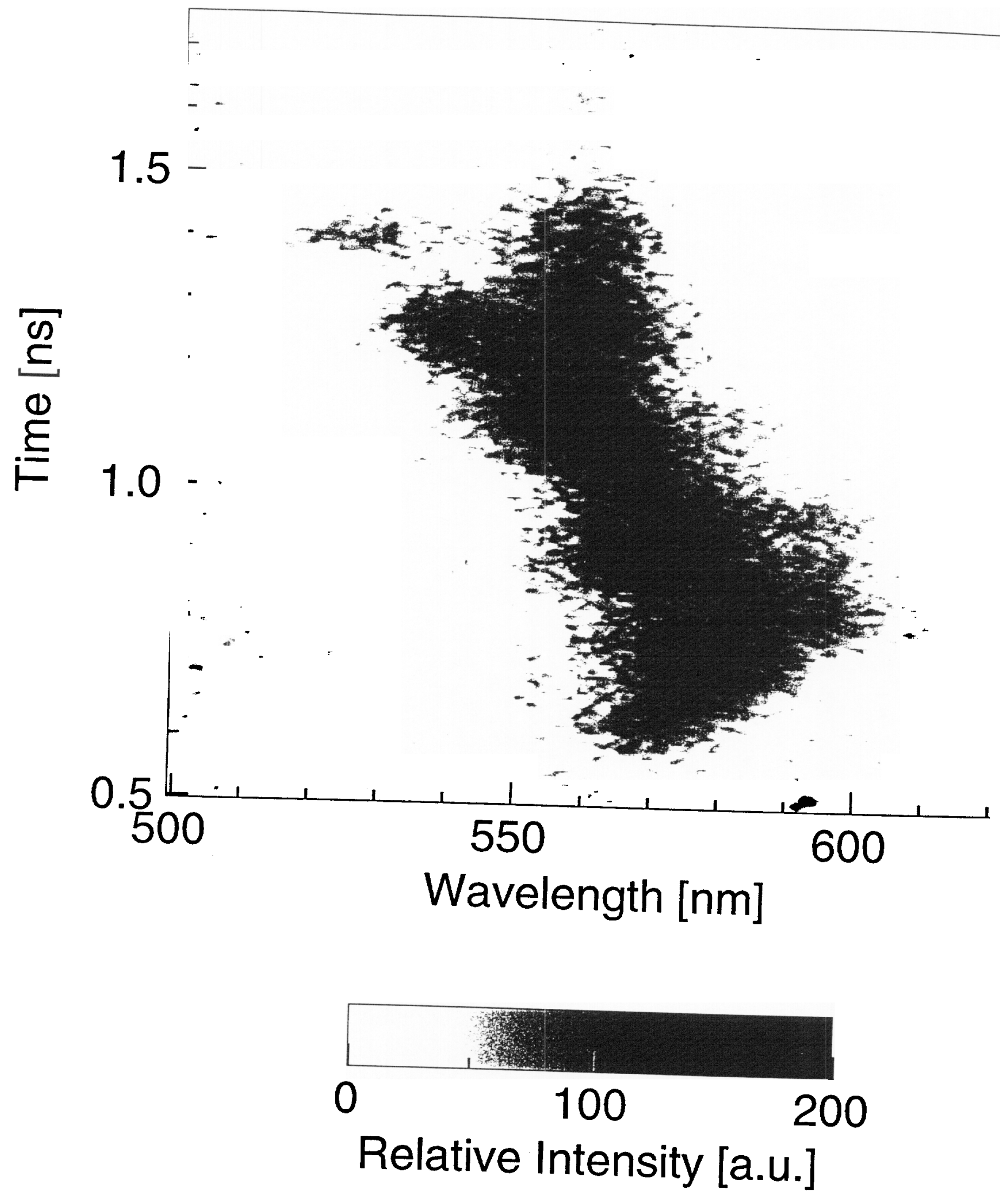

Scaled_pixel_valu_x vs. (Time_ns, Wavelength_nm ) 


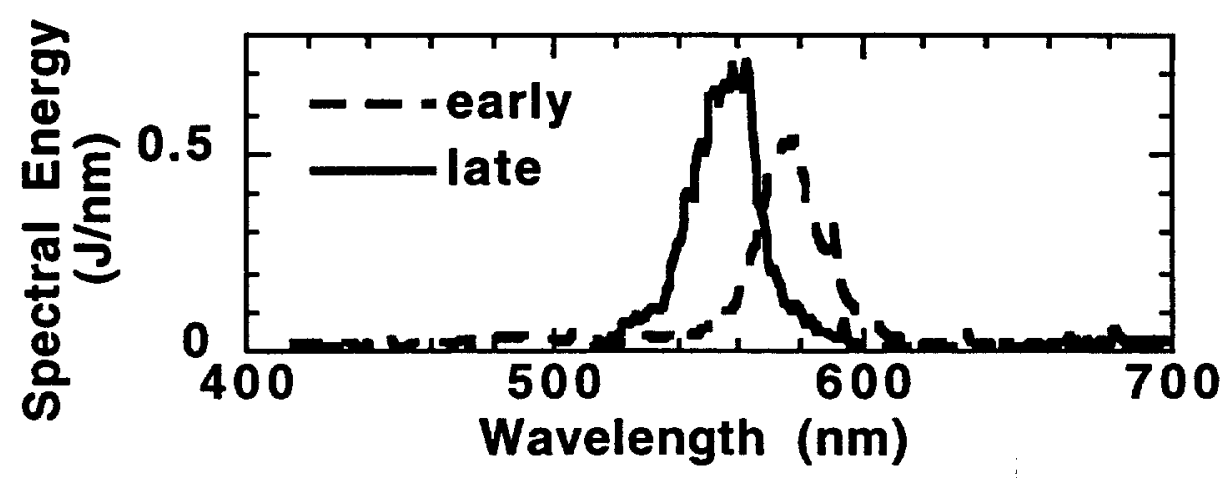

Figure 2(b)

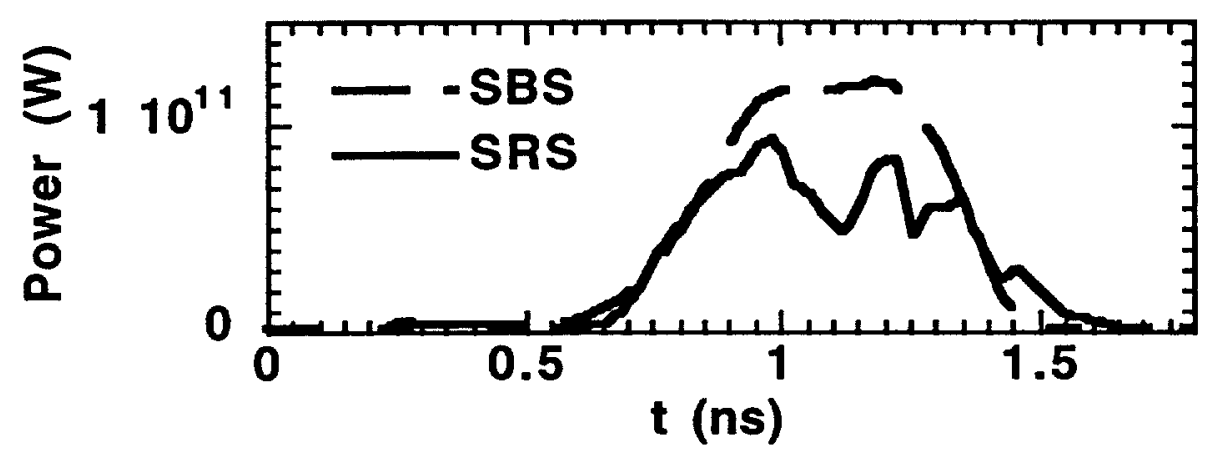

Figure 2(c) 


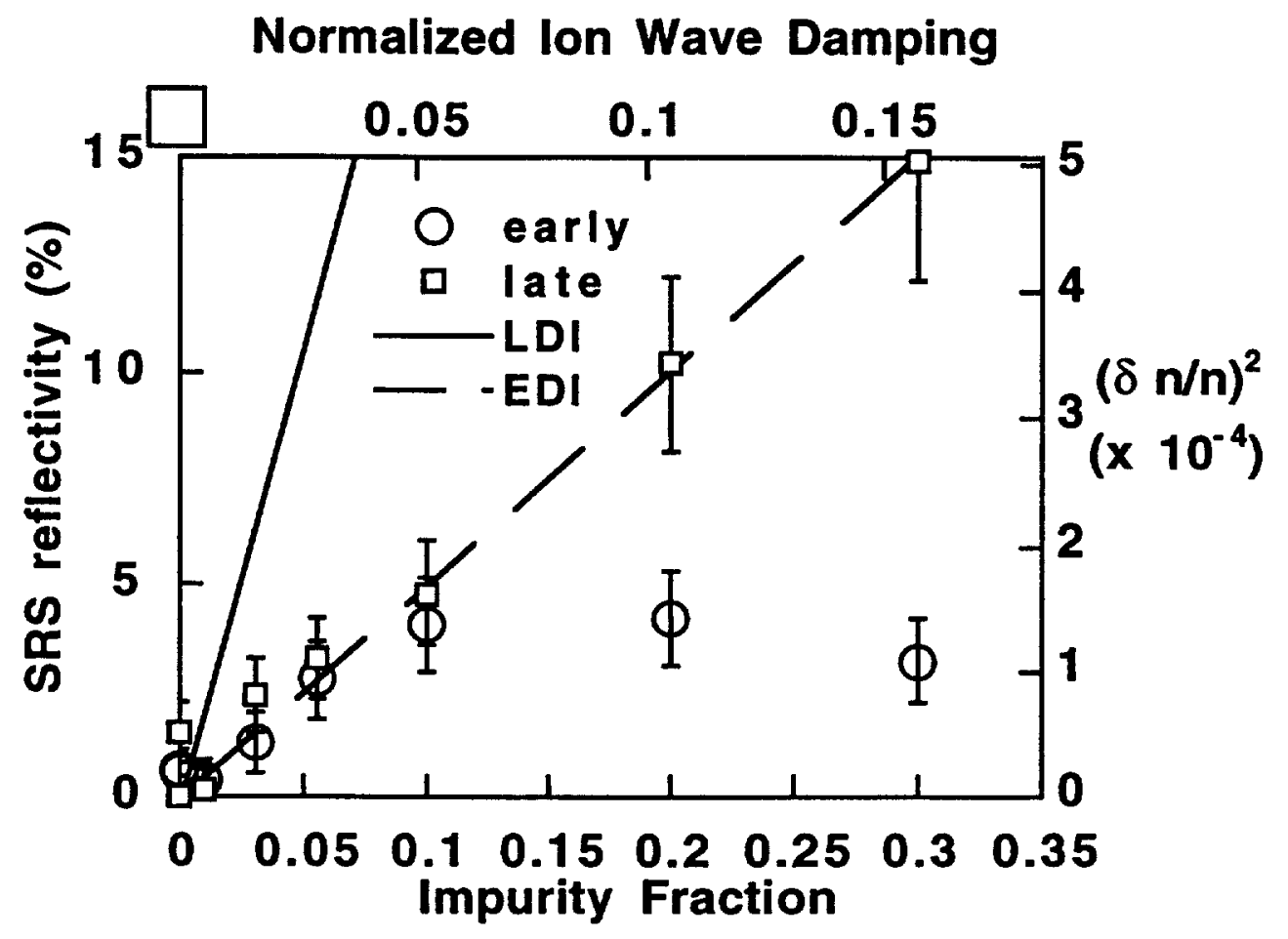

Figure 3 


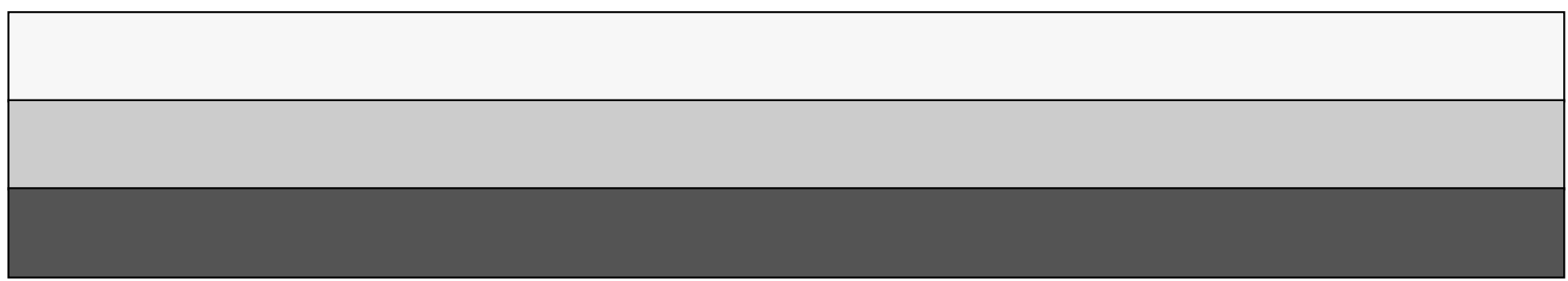

